\title{
PEDESTRIAN DEAD RECKONING USING SMARTPHONES SENSORS: AN EFFICIENT INDOOR POSITIONING SYSTEM IN COMPLEX BUILDINGS OF SMART CITIES
}

\author{
E. Saadatzadeh ${ }^{1}$, A.Chehreghan ${ }^{2}$, R. Ali Abbaspour ${ }^{3 *}$ \\ ${ }^{1}$ School of Surveying and Geospatial Engineering., College of Eng., University of Tehran., Tehran, Iran - es.saadatzadeh@ut.ac.ir \\ ${ }^{2}$ Faculty of Mining Engineering., Sahand University of Technology., Tabriz, Iran - chehreghan@sut.ac.ir \\ ${ }^{3}$ School of Surveying and Geospatial Engineering., College of Eng., University of Tehran., Tehran, Iran - abaspour@ut.ac.ir
}

\author{
Commission VI, WG VI/4
}

KEY WORDS: Indoor Positioning, Smartphone Mode Detection, Pedestrian Dead Reckoning, Smartphone Sensors, Filter

\begin{abstract}
:
This paper proposes an indoor positioning method using Pedestrian Dead Reckoning (PDR) based on the detection of the mode of the user's smartphone. In the first step, to determine the mode of carrying the smartphone (Holding, Calling, Swinging) by suitably formed feature vectors based on sensor data, three classification algorithms (Decision Tree (DT), Support Vector Machine (SVM), and KNearest Neighbor (KNN)) are evaluated. From the classification algorithm perspective, the decision tree algorithm had the best performance in terms of processing time and classification. Secondly, to determine the user position, the step detection is performed by defining the upper threshold and time threshold for Acceleration norm values. The orientation component is obtained by combining accelerometer, magnetometer, and gyroscope data using Complementary Filtering and Principal Component Analysis based on Global Acceleration (PCA-GA) methods. The mean standard deviation along the direct path for the three modes of carrying (Holding, Calling, and Swinging) were obtained 6.22, 6.82, and 14.68 degrees, respectively. Localization experiments were performed on 3 modes of carrying a smartphone in a rectangular geometry path. The mean final error of positioning from ordinary walking for the three modes of holding (Calling, Holding, Swinging) were obtained 2.11, 2.34, and 4.5 m, respectively.
\end{abstract}

\section{INTRODUCTION}

The urbanization is one of the ever increasing phenomena in the world. Therefore, the environmental, social and economic sustainability is one of the most important points for coordinating with this rapid population expansion and cities' financing. Smart City is a metropolitan area that uses various types of electronic sensors to collect and analyze the information, which is useful for managing urban assets and resources. The process involves the data collected from citizens, organizations, and urban resources that are processed and analyzed in order to assist for monitoring and managing the traffic and transportation, control, rule of information systems implementation, schools, libraries, hospitals, and the other social services. The idea of a smart city is to integrate ICT and various devices connected to the network (Internet of Things or IOT) to optimize the utilization of urban services and applications and to connect them to citizens. Smart city technology allows the authorities to interact directly with the community and the urban infrastructure and to monitor what is happening and what's going on. The integration of information technology occurs in every part of urban life to form smart cities. Some smart city applications include smart mobility, smart services, smart economy, smart buildings, smart environments, smart state, smart healthcare, smart citizens, and so on.
One of the most important issues in a smart city is the positioning and navigation in the buildings, which has attracted considerable attention among researchers.

Positioning can be carried out in open (outdoor) or closed (indoor) areas. The development of indoor positioning systems is required for indoor positioning due to reduced Global Positioning System (GPS) accuracy (Parnandi, Le et al., 2009). However, due to inaccessibility of GPS correct signals inside the building, location based services may not have sufficient accuracy and quality, although this technique is optimized for outdoor environments (Parnandi, Le et al., 2009). Therefore, establishing a reliable and accurate indoor positioning system is an emerging task. Indoor positioning is one of the significant fields of research that needs to be repeatedly revised due to the continuous development and different applications in a smart city environment. In general, indoor positioning systems are divided into two categories: infrastructure-based and infrastructure-independent. Infrastructure-based approaches such as Ultrasonic (Savvides, Han et al., 2001), Wireless Local Area Network (WLAN) (Bahl, Padmanabhan et al. 2000, Kushki, Plataniotis et al. 2007, Zhou, Yeung et al., 2008), Bluetooth, and Radio Frequency Identification (RFID) (Aalto, Göthlin et al., 2004; Baniukevic, Sabonis et al., 2011) are ways that require infrastructures such as the Internet and wireless access to point-of-access. Whereas laserbased methods (Tang, Chen et al., 2014), motion sensors (Girard,

\footnotetext{
* Corresponding author
} 
Côté et al., 2011; Nam and Engineering 2011) and visual (Mulloni, Wagner et al., 2009) are recognized as infrastructureindependent methods. RFID Based Positioning Performs based on radio frequency detection of signals related to RFID tags. The RFID method does not require a direct line of sight between the receiver and the signal transmitter. The absolute positioning of this method is within 1 to 3 meters with high power consumption [Amit et al., 2019]. In the Bluetooth-based indoor positioning method, beacons are able to position via Bluetooth using a proximity position calculation technique. At least three beacons must be installed in the building to determine the position. The beacon-related app must also be installed on the smartphone. This method has low power consumption and results in an accuracy of about 0.5 to 2 meters. There is also no need for a direct line of sight between the receiver and the transmitter [Amit et al., 2019]. The most important disadvantage of this system is the limited coverage and need to more beacons for more accurate positioning which increases the cost [Amit et al., 2019]. One of the most popular applications of WLANs is Wireless Fidelity (WiFi), estimating the location of a mobile device in its network. Time of Arrival (TOA) and Time of Differential Arrival (TODA) methods are less common in WLANs because of computational complexity and delay of time measurement. The absolute positioning accuracy of this method is about 2 to 5 meters [Amit et al., 2019]. The WiFi positioning method usually uses a Fingerprinting architecture (Kaemarungsi and Krishnamurthy 2004; Liu, Darabi et al., 2007). Received Signal Strength (RSS) information is collected from Access Points at reference points to build a database called Radio Map during the Offline phase and matched with the real time RSS collected to obtain the position on the Online phase (Kaemarungsi and Krishnamurthy 2004; Liu, Darabi et al, 2007).

Each method of indoor positioning has its own advantages and disadvantages. The choice of method will ultimately be based on accuracy, cost, ease, scalability, reliability, and security. Based on previous research, infrastructure-based methods are generally more accurate and reliable than infrastructure-independent indoor positioning methods. But infrastructure-independent approaches are superior in cost and ease due to the lack of additional infrastructure. For example, in situations such as fires where no auxiliary infrastructure such as WiFi can be used, PDR-based positioning may be the best option. On the other hand, other infrastructure-independent methods such as laser-based and imaging-based positioning lose their effectiveness in these environmental conditions. Compared to other positioning techniques, PDR can provide accurate positioning in a short period of time. Also, in this method the speed of updating the user position is faster and less energy consuming.

With the advancement of smartphones, various sensors such as accelerometer, gyroscope, magnetometer, and barometer are being added daily. Due to their popularity among the people and their small physical form and size, light weight and easy portability can be used as a great platform for indoor positioning applications. The basis of this approach is to determine the current position of the pedestrian based on their previous position, step length and walking direction (Jin, Toh et al, 2011). In fact, this method involves three steps: step detection, step length estimation, and user orientation. Since PDR positioning provides relative positions, the error during the walk will be cumulative, so many criteria such as initial point determination, direction estimation, step length estimation and number of steps affect the final positioning accuracy (Alzantot and Youssef 2012).

The purpose of this paper is to evaluate and improve the accuracy of indoor positioning using PDR-based smartphone sensors. Much research in the field of indoor positioning with PDR done or is doing. But because of the optional user movements usually, achieve the desired accuracy in this type of positioning method is difficult. Therefore, in this research, the effective components of the indoor positioning method are evaluated based on the type of carrying mode (Holding, Calling, Swinging).

The structure of this paper will be as follows. Section 2 presents the proposed system architecture. This section describes the parameters of the PDR positioning method and smartphone mode detection algorithms. The results of the mode detection and localization experiments are shown in Section 3. Finally, Section 4 describes the conclusions and future work.

\section{PROPOSED APPROACH}

The general approach of the PDR system is based on smartphone mode detection as shown in Figure 1. Using the three classification algorithms (DT, SVM, and KNN), the samples of the smartphone mode are classified and evaluated based on feature vectors from the sensor data. With mode detection, the parameters of step length estimation and orientation are adjusted based on the smartphone mode and the pedestrian position is updated. The details of the pedestrian positioning system are described in the following sections.

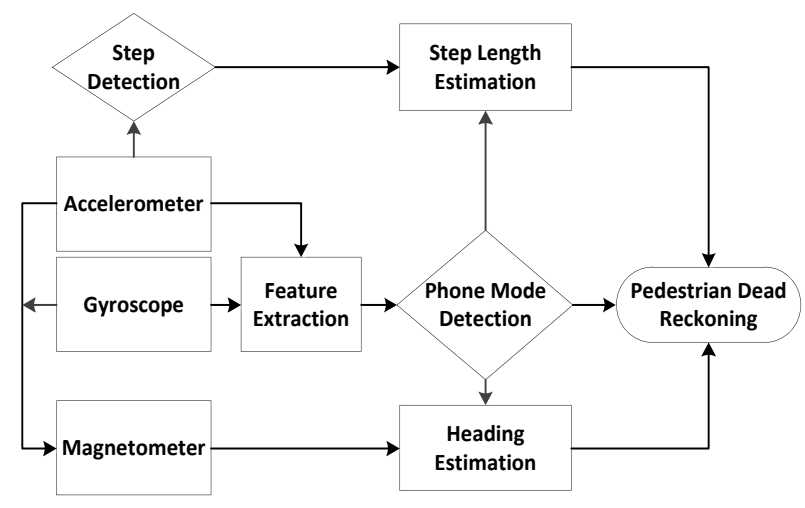

Figure 1. The architecture of the system

\subsection{PDR Positioning Method}

The PDR positioning method based on smartphone sensors determines the user's current position based on their previous position, step length and direction of movement. In general, the PDR positioning method is summarized in Equation 1:

$\left\{\begin{array}{l}\mathrm{X}_{\mathrm{k}}=\mathrm{X}_{\mathrm{k}-1}+\mathrm{L}_{\mathrm{k}-1, \mathrm{k}} * \cos \left(\text { Azimuth }_{\mathrm{k}-1, \mathrm{k}}\right) \\ \mathrm{Y}_{\mathrm{k}}=\mathrm{Y}_{\mathrm{k}-1}+\mathrm{L}_{\mathrm{k}-1, \mathrm{k}} * \sin (\text { Azimuth }\end{array}\right.$

where $\mathrm{X}, \mathrm{Y}$ are user coordinates, $\mathrm{L}$ is user step length, Azimuth is user direction angle, and $\mathrm{k}$ is step count index.

acceleration and Angular velocity data have different characteristics in different smartphone carrying modes. Therefore, based on the classification results, a flexible PDR 
algorithm is proposed to suit the smartphone carrying mode, which includes initial position estimation, step detection, step length estimation, and orientation estimation.

\subsection{Smartphone sensors}

As smartphones evolve, various sensors are added. Due to their popularity, these phones can be used as an ideal platform for indoor positioning applications. Most of these phones have different sensors of motion, orientation, and environment to determine the 3D positioning and positioning of the device. Depending on the type of sensors embedded in the smartphone, the measured raw data have different accuracy. In general, smartphone sensors can be divided into three general categories:

Motion Sensors: These sensors measure the acceleration and rotational forces along the 3 axes of the coordinate system. This category includes accelerometer, gravity, gyroscope and rotational vector sensors.

Environmental Sensors: These sensors measure various environmental parameters such as air temperature, pressure, light, and humidity. This category includes thermometers, barometers, photometers, and humidity sensors.

Position sensors: These sensors measure the physical position of the device, including magnetometers and orientation sensors.

Generally, the sensor framework uses a standard 3-axis coordinate system to express data values. For most sensors, the device coordinate system is defined by default in Figure 2.

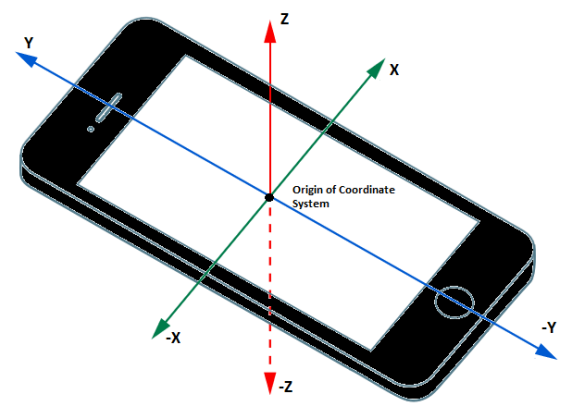

Figure 2. The device coordinate system

\subsection{Sensor data preprocessing}

This paper collects the sensor data required using the Samsung Galaxy S7 Edge. Because the PCA-GA method requires a relatively large amount of data at each step, a sampling rate of 20,000 microseconds is assumed.

Both the accelerometer sensor and the gyroscope have Offset, meaning they are not exactly zero at rest, although in theory, they should be 0 . This can be alleviated by keeping the smartphone at rest and collecting measurements over a period of time and ultimately dividing the total number of measurements. The resulting offset mean can be subtracted from all future readings. Because of the microelectromechanical structure of the sensors and the vibration of the handheld observations, the sensors embedded in the smartphone have a lot of noise in the raw signals. Therefore, a pre-processing process is applied to eliminate highfrequency noise. In this research, based on the analysis of the sensor signals in different modes of carrying the smartphone, a 3- degree Savitzky-Golay filter with a window length of 15 are used to eliminate high-frequency noise. Figure 3 and Figure 4 show the raw signals and the filtered signals of the accelerometer data and the Z-axis data of the gyroscope.

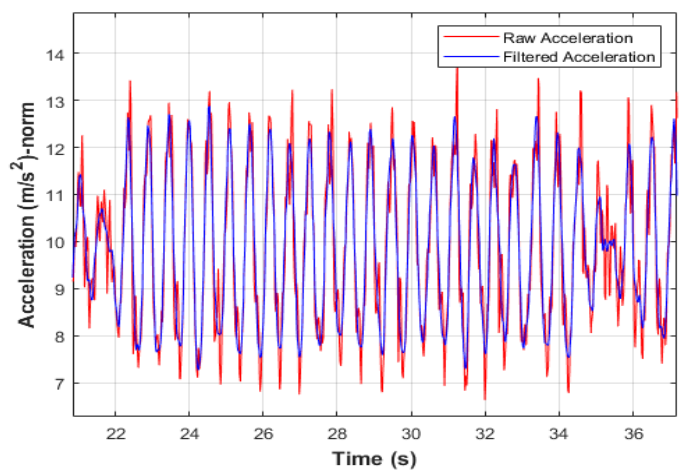

Figure 3: Raw and filtered acceleration data

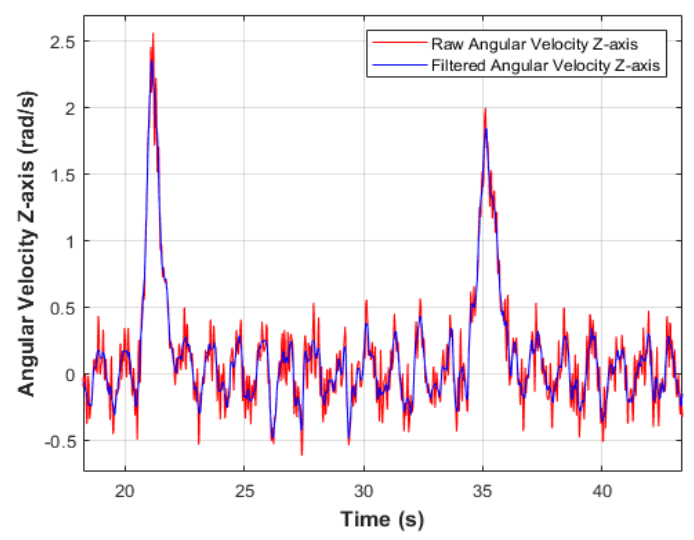

Figure 4 - Angular velocity raw and filtered data

\subsection{Experimental Analysis of the Components Affecting PDR Positioning Method}

This section describes the experimental Analysis of the effective components of the PDR positioning method based on Equation 1, which include initial position estimation, step detection, step length estimation, and direction estimation.

\subsubsection{Estimate the initial position}

The PDR method provides relative positioning, so the accuracy of initial position estimation directly affects the overall accuracy of PDR positioning. In this paper, a specific point in the experimental area is considered as the starting position or starting point.

\subsubsection{Step Detection}

User step detection is an important issue in PDR positioning based on smartphone sensors. In fact, false step detection causes an error in position estimation. In this paper, due to the periodic movement of acceleration data during the step, the acceleration data norm is used for step count. The 3-axis acceleration data of the device coordinate system in smartphone different mode has different values. By examining the 3-axis acceleration values in 
the three smartphone modes, it can be concluded that the 3 -axis acceleration norms are approximately the same. Consequently, to avoid the effect of changing the mode of carrying a smartphone, the acceleration norm according to Equation 2 is used for step detection.

$a=\sqrt{a_{x}^{2}+a_{y}^{2}+a_{z}^{2}}$

Where $\mathrm{a}_{\mathrm{x}}, \mathrm{a}_{\mathrm{y}}, \mathrm{a}_{\mathrm{z}}$ are acceleration values and $\mathrm{a}$ is acceleration norm value.

\subsubsection{Recognize volunteer peaks}

The core of the step detection algorithm is to find peaks that can represent real pedestrian steps. In this paper, an upper threshold $\lambda_{\text {thr_Up }}$ is used for filtering peaks due to the user step according to Equation 3. A time threshold is also used as $\Delta t_{\text {thr }}$ between successive peaks to consider false peaks that are less than the time threshold as false peaks. The threshold values are determined by an empirical analysis of the acceleration data in different smartphone carrying modes. Figure 5 and Figure 6 shows the detection of the candidate peaks on the acceleration norm data.

$$
\left\{\begin{array}{c}
\Delta \mathrm{T} \geq \Delta \mathrm{t}_{\text {thr }} \\
|\mathrm{a}| \geq \lambda_{\text {thr_Up }}
\end{array}\right.
$$

Where $\Delta \mathrm{T}$ is ithe nterval between two consecutive peaks, $\Delta \mathrm{t}_{\text {thr }}$ is time threshold, $\Delta \mathrm{T}$ is acceleration values, a is acceleration norm, and $\lambda_{\text {thr_Up }}$ is the upper threshold.

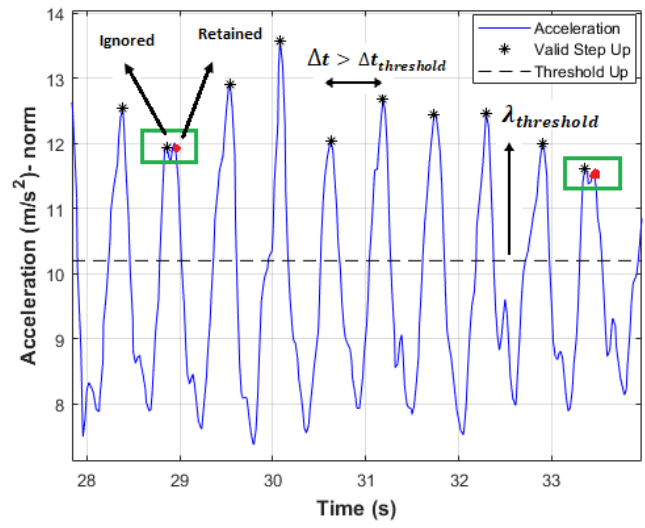

Figure 5. Detection of the valid steps for Holding

\subsubsection{Estimate step length}

The step length varies from person to person, in fact there must be a pedestrian-related variable in the step length estimation model. Also, a person's walking rate is not constant. Accordingly, assuming a constant step length value for users causes errors during positioning and excessive drift at the end of the path. In this paper, according to Equation 4 the step length is estimated based on a linear combination of the step frequency and the variance of the norm acceleration of each step (Ladetto, 2000).

$\mathrm{L}_{\mathrm{k}-1, \mathrm{k}}=\mathrm{A}+\mathrm{B} \times \mathrm{f}_{\mathrm{k}}+\mathrm{C} \times \sigma_{\mathrm{k}}^{2}$ where $L$ is step length, $f_{k}$ is step frequency, $\sigma_{k}^{2}$ is acceleration norm variance in one step, and $\mathrm{A}, \mathrm{B}, \mathrm{C}$ are experimental coefficients.

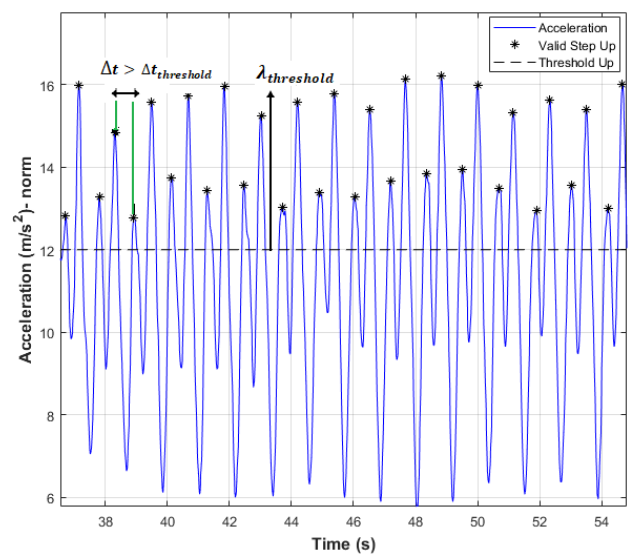

Figure 6. Detection of the valid steps for Swinging

\subsubsection{Determine User's Orientation}

The calculation of pedestrian orientation angles greatly affects the accuracy of the positioning system but is also the most difficult part of the PDR. Since for Holding and Calling do not change the orientation of the smartphone much towards the body, the pedestrian orientation can be determined by removing the offset orientation. For Swinging mode where the smartphone position is constantly changing and offset orientation is not fixed, a PCAbased approach with global acceleration (Wang, Liu et al., 2018) for pedestrian orientation evaluation is provided. Figure 7 shows the direction estimation method for each of the smartphone carrying mode.

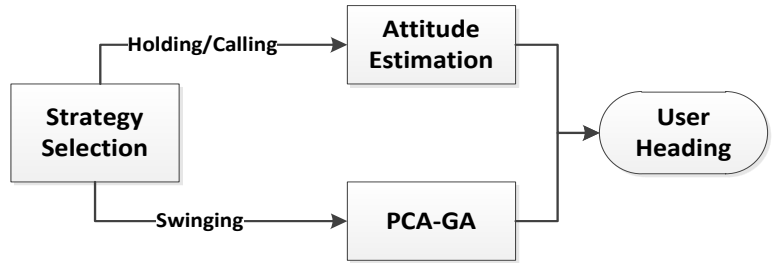

Figure 7. Flow chart of the user heading estimation approach

\subsubsection{Complimentary Filter Method for Holding and Calling}

Using trigonometry, an estimate of Pitch (rotation about Y-axis) and Roll (rotation about $\mathrm{X}$-axis) angles is calculated using the mean of gravity acceleration data at each step according to Equation 5 and Equation 6.

$\widehat{\varphi}_{\mathrm{Roll}}=\tan ^{-1} \frac{\overline{\mathrm{g}}_{\mathrm{y}}}{\sqrt{\overline{\mathrm{g}}_{\mathrm{x}}^{2}+\overline{\mathrm{g}}_{\mathrm{z}}^{2}}}$

$\widehat{\theta}_{\text {Pitch }}=\tan ^{-1} \frac{\overline{\mathrm{g}}_{\mathrm{x}}}{\sqrt{\overline{\mathrm{g}}_{\mathrm{y}}^{2}+\overline{\mathrm{g}}_{\mathrm{z}}^{2}}}$

where $\bar{g}_{x}, \bar{g}_{y}, \bar{g}_{z}$ are mean of gravity acceleration data over a onestep interval, $\widehat{\varphi}_{\text {Roll,Acc }}$ is rotation about X-axis, and $\hat{\theta}_{\text {Pitch,Acc }}$ is rotation about $\mathrm{Y}$-axis. 
The gyroscope measures the angular velocity within the body. In order to obtain the changes in the inertial framework, the state transfer matrix in (Philip, 2019) is used according to Equation 7.

$$
\left[\begin{array}{c}
\varphi_{\text {Roll }} \\
\theta_{\text {Pitch }} \\
\psi_{\text {Yaw }}
\end{array}\right]=\left[\begin{array}{ccc}
1 & \sin (\varphi) * \tan (\theta) & \cos (\varphi) * \tan (\theta) \\
0 & \cos (\varphi) & -\sin (\varphi) \\
0 & \sin (\varphi) * \sec (\theta) & \cos (\varphi) * \sec (\theta)
\end{array}\right]\left[\begin{array}{l}
\bar{W} \mathrm{x} \\
\bar{W} \mathrm{y} \\
\overline{\mathrm{W}} \mathrm{z}
\end{array}\right] \mathrm{dt}
$$

where $\varphi, \theta, \theta$ are Euler angle from gyroscope data, $\bar{W} x, \bar{W} y, \bar{W} z$ are mean of gyroscope data over a one-step interval, and $d t$ is a one-step interval.

The combination of the two estimates is performed to obtain the optimal estimate. The final result is according to Equation 8.

$\varphi_{\mathrm{k}+1}=(1-\alpha) *\left(\varphi_{\mathrm{k}}+\varphi_{\mathrm{gyro}}\right)+\alpha * \widehat{\varphi}_{\mathrm{Acc}}$

where $\varphi$ is the roll angle, $\widehat{\varphi}_{\text {Acc }}$ is roll angle from acceleration data, $\varphi_{\text {gyro }}$ is roll angle from angular velocity data, $\alpha$ is a constant, and $\mathrm{k}$ is step count index.

$\alpha$ is a constant between 0 and 1. The larger $\alpha$ indicates more confidence in the accelerometer measurements. As $\alpha$ approaches 0 , the final value relies more on gyroscope measurements. In this paper, the $\alpha$ value is set to 0.1 due to the high accuracy of the gyroscope data. By obtaining the angular values of Pitch and Roll, the absolute azimuth value is obtained using magnetic induction in the $\mathrm{X}$ and $\mathrm{Y}$ directions according to Equation 9.

Azimuth $_{\text {mag }}=\tan ^{-1} \frac{\overline{\mathrm{m}}_{\mathrm{y}}}{\overline{\mathrm{m}}_{\mathrm{x}}}$

Where $\overline{\mathrm{m}}_{\mathrm{x}}, \overline{\mathrm{m}}_{\mathrm{y}}, \overline{\mathrm{m}}_{\mathrm{z}}$ are mean magnetic values over a one-step interval, and Azimuth ${ }_{\text {mag }}$ is smartphone orientation.

In the non-horizontal state, the horizontal values of $\mathrm{m}_{\mathrm{x}}^{\mathrm{h}}$ and $\mathrm{m}_{\mathrm{y}}^{\mathrm{h}}$ are obtained by Equation 10 (Moafipoor et al., 2007).

$\left[\begin{array}{c}\mathrm{m}_{\mathrm{x}}^{\mathrm{h}} \\ \mathrm{m}_{\mathrm{y}}^{\mathrm{h}}\end{array}\right]=\left[\begin{array}{c}\overline{\mathrm{m}}_{\mathrm{x}} * \cos (\varphi)+\overline{\mathrm{m}}_{\mathrm{y}} * \sin (\theta)-\overline{\mathrm{m}}_{\mathrm{z}} * \cos (\theta) * \sin (\varphi) \\ \overline{\mathrm{m}}_{\mathrm{y}} * \cos (\theta)+\overline{\mathrm{m}}_{\mathrm{z}} * \sin (\theta)\end{array}\right]$

where $\varphi, \theta, \theta$ are Euler angle from gyroscope data, $\overline{\mathrm{m}}_{\mathrm{x}}, \overline{\mathrm{m}}_{\mathrm{y}}, \overline{\mathrm{m}}_{\mathrm{z}}$ are mean magnetic values over a one-step interval, and $\mathrm{m}_{\mathrm{x}}^{\mathrm{h}}, \mathrm{m}_{\mathrm{y}}^{\mathrm{h}}$ are horizontal values of $\mathrm{m}_{\mathrm{x}}^{\mathrm{h}}$ and $\mathrm{m}_{\mathrm{y}}^{\mathrm{h}}$.

\subsubsection{PCA-GA Method for Swinging mode}

From the observations, It can be seen that during the walk, the acceleration changes in $\mathrm{X}$-axis of the pedestrian coordinate system are minimal. The pedestrian coordinate system is depicted in Figure 8, where $\mathrm{Y}$-axis points to the direction of pedestrians walking, $\mathrm{X}$-axis points to the right side of pedestrian's body and Z-axis points to the opposite direction of gravity (Wang, Liu et al. 2018). Therefore, the direction of the third eigenvector in PACGA is $\mathrm{R}=\left[\mathrm{r}_{\mathrm{x}} \cdot \mathrm{r}_{\mathrm{y}} \cdot \mathrm{r}_{\mathrm{z}}\right]$, which is perpendicular to the pedestrian's direction on the horizontal plane (Wang, Liu et al. 2018).

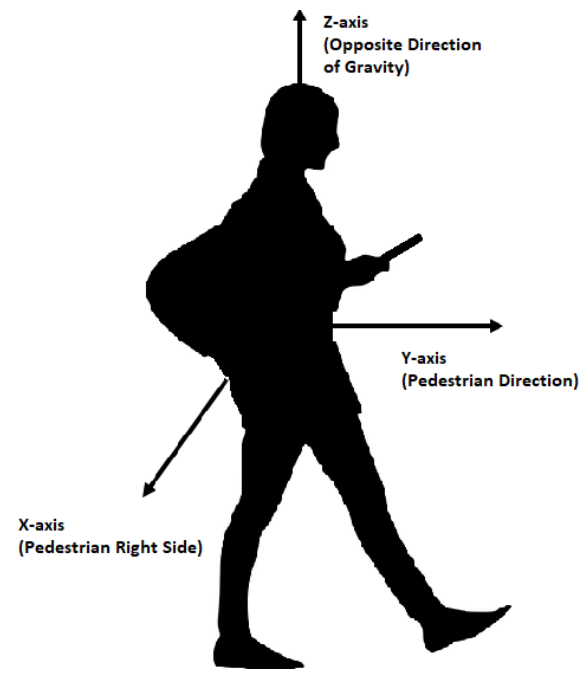

Figure 8 . The illustration of the pedestrian coordinate system

The extracted eigenvectors from the global accelerations of device coordinate system are shown in Figure 9. The vector right has the problem of 180 ambiguity for each step (Kunze, Lukowicz et al., 2009), R may be the right or left of the body. To solve this ambiguity, the orientations of the acceleration values are analyzed as the smartphone Swinging in different hands. According to the results of the analysis, the right $\mathrm{R}$-value is proportional to the user coordinate system. For example, when the user carries the smartphone in Swinging mode on the right hand side of his body, the $\mathrm{Z}$ axis of the device coordinate system is almost to the left of the body, so the $r_{z}$ components of the $R$ vector must be negative for each step.

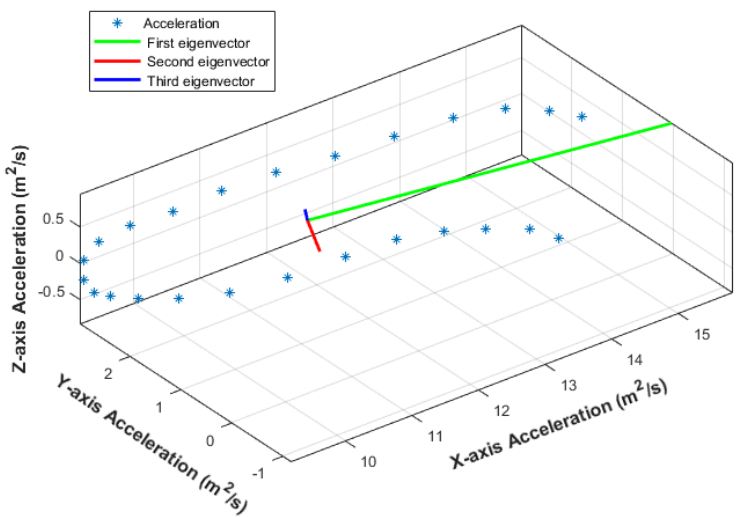

Figure 9. The eigenvectors extraction for Swinging

Next, the R-vector is plotted on the horizontal plane according to Equation 11 using the gravitational vector (Mizell 2003).

$G=-\left(\bar{g}_{x}, \bar{g}_{y}, \bar{g}_{z}\right), \quad R_{h}=R-\left(\frac{R \cdot G}{\text { G.G }}\right) G$

where $R$ is vector right, $R_{h}$ is horizontal vector right, and $G$ is mean of gravity acceleration data over a one-step interval.

The gravitational vector $\mathrm{G}$ points downward and the magnetic vector $\mathrm{M}$ points north. The east vector $\mathrm{E}$ is obtained by Equation 12 by multiplying the two magnetic and gravity vectors in the system coordinate system (Kamisaka, Muramatsu et al., 2011). 
$\mathrm{M}=\left(\overline{\mathrm{m}}_{\mathrm{x}}, \overline{\mathrm{m}}_{\mathrm{y}}, \overline{\mathrm{m}}_{\mathrm{z}}\right) \quad, \quad \mathrm{E}=\mathrm{G} \times \mathrm{M}$

where $\mathrm{M}$ is mean magnetic values over a one-step interval, $\mathrm{G}$ is mean of gravity acceleration data over a one-step interval, and $\mathrm{E}$ is east vector.

Finally, the angle of user orientation is calculated by Scalar Product between the east vector and the horizontal vector by the Equation 13.

Azimuth $=\cos ^{-1}\left(\frac{\mathrm{R}_{\mathrm{h}} \cdot \mathrm{E}}{\left|\mathrm{R}_{\mathrm{h}}\right| \mathrm{E} \mid}\right)$

where $\mathrm{R}_{\mathrm{h}}$ is horizontal vector right, $\mathrm{E}$ is east vector, and Azimuth is user orientation.

\subsection{Smartphone Mode Classification}

Smartphone mode is optional in the navigation process, so the accelerometer and gyroscope signals have different shapes and values. For this reason, this paper uses accelerometer and gyroscope sensors to detect smartphone mode. The classification algorithm consists of feature extraction and smartphone mode detection. Carrying modes according to Figure 10 include Holding, Calling, Swinging. The mean values of the acceleration and variance values of the Angular Velocity for each step are shown in Figure 11 and Figure 12, respectively.

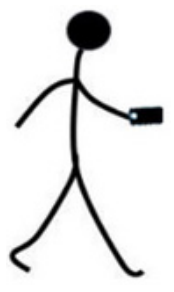

(a)

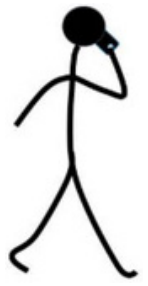

(b)

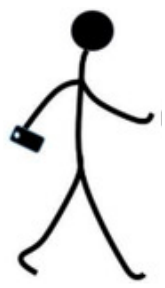

(c)
Figure 10. Three smartphone modes (a) Holding, (b) Calling, (c) Swinging

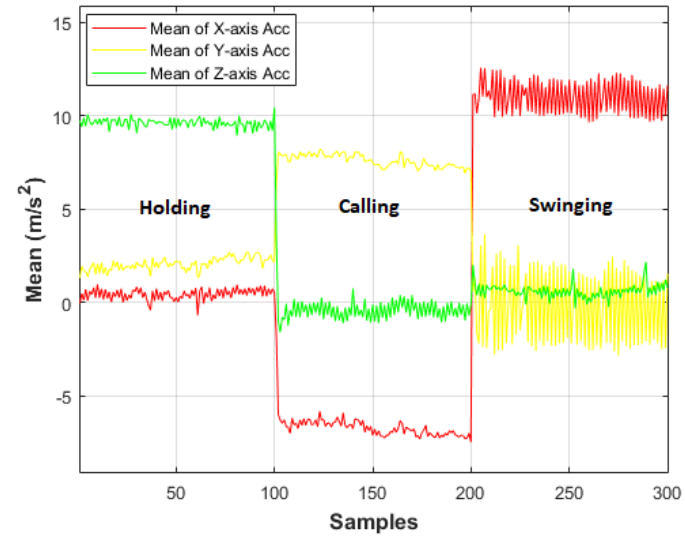

Figure 11. The mean of accelerations with three smartphone modes while pedestrian is walking

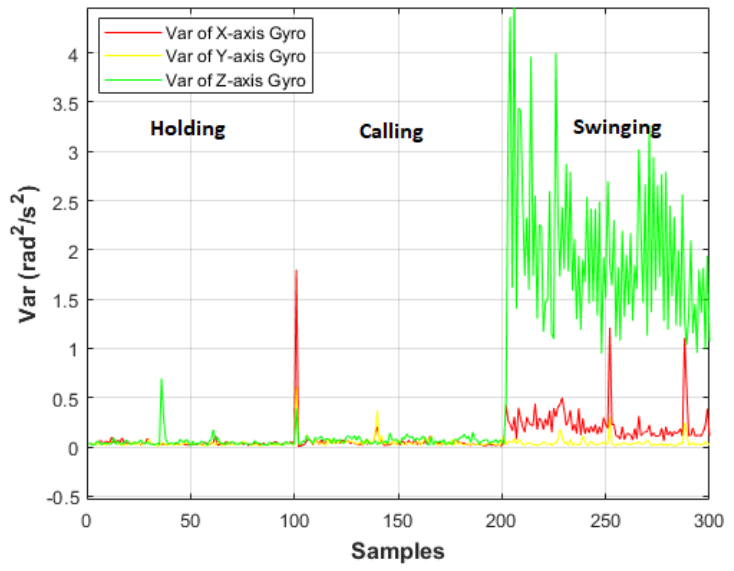

Figure 12. The variances of angular velocities with three smartphone modes while the pedestrian is walking

\subsubsection{Extract and Select Features}

The filtered data is inadequate to detect the smartphone carrying mode. In fact, the feature information extracted from the acceleration and angular velocity values in the step-by-step time windows are needed. The statistical features of the sensor signals include the mean and variance of the acceleration and angular velocity values obtained over a $50 \%$ coverage period over the next step. In this paper, the time domain features vector $F$ is summarized as follows:

$\mathrm{F}=\left[\mathrm{M}_{\mathrm{a}}, \mathrm{V}_{\mathrm{a}}, \mathrm{M}_{\mathrm{w}}, \mathrm{V}_{\mathrm{w}}\right]$

where $M_{a}$ is mean of three-direction acceleration values per step, $V_{a}$ is a variance of three-direction acceleration values per step, $\mathrm{M}_{\mathrm{w}}$ is mean of three-direction angular velocity values per step, and $V_{w}$ is the variance of three-direction angular velocity values per step.

\subsubsection{Classification}

In this paper, using the extracted features in Section 2-4-1 from three classification algorithms (DT, SVM, and KNN) are used to classify the 3 modes of smartphone carrying.

\section{IMPLEMENTATION AND EVALUATION OF RESULTS}

In this section, experiments are presented to evaluate the performance of the proposed methods of smartphone mode detection and indoor positioning. The smartphone used in this experiment is a Samsung Galaxy S7 Edge with a delay time of 20,000 microseconds. Required sensor data is stored continuously by creating a file in the smartphone memory as the data is read.

\subsection{Smartphone Mode Detection Test}

Participants walked in different modes of carriage and the data was labeled according to the mode of carriage. For each class, 250 samples were obtained using the corresponding data. $70 \%$ of the feature data were selected as training data set and the remaining $30 \%$ for testing and evaluation. Based on the DT, SVM, and KNN learning algorithms, the classification of smartphone mode is performed. Confusion matrices of all 3 algorithms are created according to Tables 1 to 3 to better analyze the results. In the 
Confusion matrix, the rows represent instances of the actual smartphone mode and the columns represent instances of the detected smartphone mode.

\begin{tabular}{|c|c|c|c|}
\hline DT & Holding & Calling & Swinging \\
\hline Holding & 250 & 0 & 0 \\
\hline Calling & 1 & 249 & 0 \\
\hline Swinging & 2 & 0 & 248 \\
\hline
\end{tabular}

Table 1. The confusion matrix for smartphone modes detection DT.

\begin{tabular}{|c|c|c|c|}
\hline SVM & Holding & Calling & Swinging \\
\hline Holding & 250 & 1 & 0 \\
\hline Calling & 2 & 248 & 0 \\
\hline Swinging & 1 & 0 & 249 \\
\hline
\end{tabular}

Table 2. The confusion matrix for smartphone modes detection by SVM.

\begin{tabular}{|c|c|c|c|}
\hline KNN & Holding & Calling & Swinging \\
\hline Holding & 249 & 1 & 0 \\
\hline Calling & 2 & 248 & 0 \\
\hline Swinging & 1 & 0 & 249 \\
\hline
\end{tabular}

Table 3. The confusion matrix for smartphone modes detection by KNN

\begin{tabular}{|c|c|c|}
\hline $\begin{array}{c}\text { Classification } \\
\text { Algorithms }\end{array}$ & $\begin{array}{c}\text { Mean } \\
\text { accuracy }\end{array}$ & Time (s) \\
\hline DT & $\% 99.6$ & 1.39 \\
\hline SVM & $\% 99.6$ & 14 \\
\hline KNN & $\% 99.5$ & 1.45 \\
\hline
\end{tabular}

Table 4. Time and accuracy of detection classification algorithms

According to Table 4, the results show that the classification accuracy of all three algorithms is nearly identical. The DT algorithm has less detection time and a smaller model size. Therefore, due to the importance of timing of mode detection and simplicity of computation, it is selected as the most optimal algorithm for detecting the mode of carrying the smartphone.

\subsection{Localization experiment}

Participants walked along a rectangular path (width is 22 and length is 33 meters) in 3 modes of carrying the smartphone. The step detection results and path length estimates are listed in Table 5 and Table 6 , respectively.

\begin{tabular}{|c|c|c|c|}
\hline Mode & $\begin{array}{c}\text { Actual } \\
\text { number }\end{array}$ & $\begin{array}{c}\text { Mean of } \\
\text { detection } \\
\text { number }\end{array}$ & accuracy \\
\hline Holding & 125 & 124 & $\% 99$ \\
\hline Calling & 125 & 124 & $\% 99$ \\
\hline Swinging & 125 & 123 & $\% 99$ \\
\hline
\end{tabular}

Table 5. Results of the step detection algorithm

\begin{tabular}{|c|c|c|c|}
\hline Mode & $\begin{array}{c}\text { Actual } \\
\text { length (m) }\end{array}$ & $\begin{array}{c}\text { Mean } \\
\text { estimated } \\
\text { Length (m) }\end{array}$ & Error (\%) \\
\hline Holding & 110 & 108.79 & 1.1 \\
\hline Calling & 110 & 108.6 & 1.3 \\
\hline Swinging & 110 & 107.8 & 2 \\
\hline
\end{tabular}

Table 6 . Results of the estimated path length algorithm

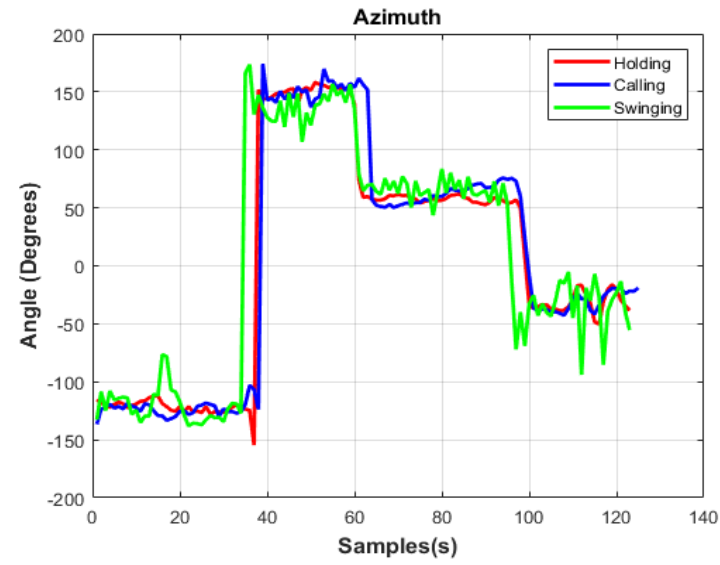

Figure 13. the experimental path direction estimation for the three modes of carrying of the smartphone.

Figure 13 shows the experimental path direction estimation for the three modes of carrying of the smartphone. The mean standard deviations of the direction estimates along the straight path for the Holding, Calling and Swinging modes were 6.22, 6.82 and 14.68, respectively. In fact, the swing of the smartphone in the third mode causes more standard deviation.

The localization experiment was performed in a rectangular path in the building of the Tehran University Football Hall of Fame. The bold black line represents the true pedestrian path and its total length is 110 meters. Markers were used along the path to guide the path. Participants began walking along the path with 3 modes of carriage and returned to the starting point at the end of the movement to assess the final positioning accuracy. Localization experiments Were done on the horizontal surface of the land, regardless of altitude change at the floor level.

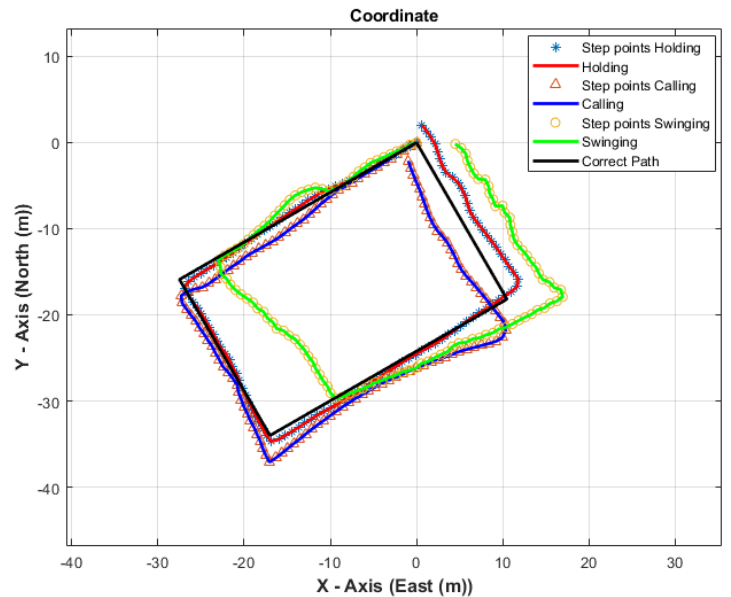

Figure 14 - Estimated paths of 3 modes of carrying a smartphone during the walk

Figure 14 shows the estimated paths of 3 modes of carrying the smartphone during walking. Offset variability between the direction of the smartphone and the direction of movement of the user can deviate from the actual path. Since the smartphone mode in Holding and Calling mode is almost stable and in Swinging mode, there is a sharp swing, the mean positioning error for the experimental path for the 3 modes is $2.11,2.34$ and $4.5 \mathrm{~m}$, respectively. 


\section{CONCLUSIONS AND FUTURE WORK}

This paper proposes an indoor positioning method based on the detection of the type of smartphone carriage. In the first step, based on 12 feature vectors from accelerometer and gyroscope sensor data, decision tree (DT), Support Vector Machine (SVM) and K-Nearest Neighbor (KNN) algorithms were evaluated to detect the mode of carrying the smartphone. From the state detection perspective, due to the small size of the model, simplicity of calculation and less detection time, the DT algorithm was chosen as the most optimal algorithm. In the second step, the step detection parameters and the step length estimation were determined based on the smartphone mode. Step detection was performed using the upper threshold and a time threshold for identifying volunteer peaks. The step length estimation process was also performed with the parameters of the step length estimation model. The orientation component was obtained by combining acceleration, gyroscope and magnetic data using complementary filtering and PCA-GA methods. The complementary filter method was used for both Holding and Calling modes that did not significantly change the orientation of the smartphone to the user body. In Swinging Carry mode, where smartphone mode and offset orientation are constantly changing, a PCA-GA method was used. The mean standard deviation along the straight path for Holding, Calling and Swinging modes were $6.22,6.82$ and 14.68 , respectively. Localization experiments were performed in 3 smartphone modes in a rectangular geometry path. The mean final error of the normal walk for the three modes (Holding, Calling, Swinging) were 2.11, 2.34 and $4.5 \mathrm{~m}$, respectively.One of the main advantages of this research is that it is not dependent on any external infrastructure. indoor positioning of the user in three modes of carrying is another advantage that improves system performance. In this way, the speed of updating the user position is faster and the power consumption is lower. But one of the disadvantages that affect the final accuracy of the proposed system is the lack of calibration of magnetic data, which is time consuming and difficult. In future work, we will develop the system by combining different user movement modes and smartphone modes. By using a barometer sensor and step counting on the stairway, precise elevation changes can be used to determine the building floor. We can also use Map Matching to increase the accuracy of position estimation and correction of false positions.

\section{REFERENCES}

Aalto, L., N. Göthlin, J. Korhonen and T. Ojala (2004). Bluetooth and WAP push based location-aware mobile advertising system. Proceedings of the 2nd international conference on Mobile systems, applications, and services, ACM.

Alzantot, M. and M. Youssef (2012). UPTIME: Ubiquitous pedestrian tracking using mobile phones. 2012 IEEE Wireless Communications and Networking Conference (WCNC), IEEE.

Bahl, P., V. N. Padmanabhan, V. Bahl and V. Padmanabhan (2000). "RADAR: An in-building RF-based user location and tracking system."

Baniukevic, A., D. Sabonis, C. S. Jensen and H. Lu (2011). Improving wi-fi based indoor positioning using bluetooth addons. 2011 IEEE 12th International Conference on Mobile Data Management, IEEE.

Girard, G., S. Côté, S. Zlatanova, Y. Barette, J. St-Pierre and P. J. S. Van Oosterom (2011). "Indoor pedestrian navigation using foot-mounted IMU and portable ultrasound range sensors." 11(8): 7606-7624.

Jin, Y., H.-S. Toh, W.-S. Soh and W.-C. Wong (2011). A robust dead-reckoning pedestrian tracking system with low cost sensors. 2011 IEEE International Conference on Pervasive Computing and Communications (PerCom), IEEE.

Kaemarungsi, K. and P. Krishnamurthy (2004). Modeling of indoor positioning systems based on location fingerprinting. Ieee Infocom 2004, IEEE.

Kamisaka, D., S. Muramatsu, T. Iwamoto, H. J. I. t. o. i. Yokoyama and systems (2011). "Design and implementation of pedestrian dead reckoning system on a mobile phone." 94(6): 1137-1146.

Kunze, K., P. Lukowicz, K. Partridge and B. Begole (2009). Which way am I facing: Inferring horizontal device orientation from an accelerometer signal. 2009 International Symposium on Wearable Computers, IEEE.

Kushki, A., K. N. Plataniotis and A. N. J. I. t. o. m. c. Venetsanopoulos (2007). "Kernel-based positioning in wireless local area networks." 6(6): 689-705.

Ladetto, Q. (2000). On foot navigation: continuous step calibration using both complementary recursive prediction and adaptive Kalman filtering. Proceedings of ION GPS

Amit, K., Ajit, G,. Subho, B,. (2019). https://www.inertialelements.com.

Liu, H., H. Darabi, P. Banerjee, J. J. I. T. o. S. Liu, Man, and P. C. Cybernetics (2007). "Survey of wireless indoor positioning techniques and systems." 37(6): 1067-1080.

Mizell, D. (2003). Using gravity to estimate accelerometer orientation. null, Citeseer.

Moafipoor, S., D. A. Grejner-Brzezinska and C. Toth (2007). Adaptive calibration of a magnetometer compass for a personal navigation system. IGNSS Symposium.

Mulloni, A., D. Wagner, I. Barakonyi and D. J. I. P. C. Schmalstieg (2009). "Indoor positioning and navigation with camera phones." 8(2): 22-31.

Nam, Y. J. J. o. I. S. and Engineering (2011). "Map-based indoor people localization using an inertial measurement unit." 27(4): 1233-1248.

Parnandi, A., K. Le, P. Vaghela, A. Kolli, K. Dantu, S. Poduri and G. S. Sukhatme (2009). Coarse in-building localization with smartphones. International Conference on Mobile Computing, Applications, and Services, Springer.

Savvides, A., C.-C. Han and M. B. Strivastava (2001). Dynamic fine-grained localization in ad-hoc networks of sensors. Proceedings of the 7th annual international conference on Mobile computing and networking, ACM.

Tang, J., Y. Chen, A. Jaakkola, J. Liu, J. Hyyppä and H. J. S. Hyyppä (2014). "NAVIS-An UGV indoor positioning system using laser scan matching for large-area real-time applications." 14(7): 11805-11824.

Wang, B., X. Liu, B. Yu, R. Jia and X. J. S. Gan (2018). "Pedestrian dead reckoning based on motion mode recognition using a smartphone." 18(6): 1811.

Philip (2019). http://philsal.co.uk/projects/imu-attitude estimation

Zhou, J., W. M.-C. Yeung and J. K.-Y. Ng (2008). Enhancing indoor positioning accuracy by utilizing signals from both the mobile phone network and the wireless local area network. 22nd International Conference on Advanced Information Networking and Applications (aina 2008), IEEE. 